J. Lake Sci. (湖泊科学), 2020, 32(3): 597-608

DOI 10. 18307/2020. 0301

(C) 2020 by Journal of Lake Sciences

\title{
全球变化下青藏高原湖泊在地表水循环中的作用”
}

\author{
朱立平 ${ }^{1,2,5 * *}$, 彭 萍 ${ }^{1}$, 张国庆 ${ }^{1,2}$,乔宝晋 ${ }^{3}$, 刘 羽中 ${ }^{1,5}$,杨瑞敏 ${ }^{4}$, 王君波 ${ }^{1,2}$ \\ (1: 中国科学院青藏高原研究所青藏高原环境与地表过程重点实验室, 北京 100101) \\ (2: 中国科学院青藏高原地球科学创新卓越中心,北京 100101) \\ (3: 郑州大学地球科学与技术学院,郑州 450001) \\ (4:兰州大学资源环境学院,兰州 730000$)$ \\ (5: 中国科学院大学, 北京 100049)
}

摘 要: 青藏高原是地球上最重要的高海拔地区之一,对全球变化具有敏感响应. 青藏高原作为 “亚洲水塔”,其地表水 资源及其变化对高原本身及周边地区的经济社会发展具有重要的影响. 然而, 在气候变暖的情况下,构成高原地表水资 源的各个组分, 如冰川、湖泊、河流、降水等水体的相变及其转化却鲜为人知. 湖泊是青藏高原地表水体相变和水循环的 关键环节. 湖泊面积、水位和水量对西风和印度季风的降水变化非常敏感,但湖泊面积和水量变化在不同区域和时段的 响应也不尽相同. 湖泊水温对气候变暖具有明显响应, 湖泊水温和水下温跃层深度的变化能够对水一气的热量交换具有 明显影响, 从而影响了区域蒸发和降水等水循环过程. 由于湖泊水量增加, 高原中部色林错地区湖泊盐度自 1970s 以来普 遍下降. 根据 60 多个湖泊实地监测建立的遥感反演模型研究发现, 2000-2019 年湖泊透明度普遍升高. 对不同补给类型 的大湖水量平衡监测发现, 影响湖泊变化的气象和水文要素具有较大差异. 在目前的暖湿气候条件下, 青藏高原的湖泊 将会持续扩张. 为了深人认识湖泊变化在青藏高原区域水循环和气候变化中的作用,需要全面了解湖泊水量赋存及连续 的时间序列变化,需要深人了解湖泊理化参数变化及对湖泊一大气之间热量交换的影响,需要更多来自大湖流域的综合 连续观测数据.

关键词: 青藏高原; 湖泊面积和水量;水量平衡; 理化参数; 调查与观测

\section{The role of Tibetan Plateau lakes in surface water cycle under global changes *}

ZHU Liping $^{1,2,5 * *}$, PENG Ping ${ }^{1}$, ZHANG Guoqing ${ }^{1,2}$, QIAO Baojin ${ }^{3}$, LIU Chong ${ }^{1,5}$, YANG Ruimin ${ }^{4} \&$ WANG Junbo ${ }^{1,2}$

(1: Key Laboratory of Tibetan Plateau Environment and Landform Process, Institute of Tibetan Plateau Research, Chinese Academy of Sciences, Beijing 100101, P.R.China)

(2: CAS Center for Excellence in Tibetan Plateau Earth Sciences, Beijing 100101, P.R.China)

(3: School of Geoscience and Technology, Zhengzhou University, Zhengzhou 450001, P.R. China)

(4: College of Resources and Environment, Lanzhou University, Lanzhou 730000, P.R.China)

(5: University of Chinese Academy of Sciences, Beijing 100049, P.R.China)

Abstract: The Tibetan Plateau is one of the most important high altitude areas on the earth, which is sensitive to global changes. As the Asia Water Tower, the land surface water resources and their changes of the Tibetan Plateau have an important impact on the economic and social development of the plateau itself and its surrounding areas. However, under the condition of climate warming, there is less recognizance of the surface water facies shifting and their transformation for components of surface water resources, such as glaciers, lakes, rivers, precipitation and other water bodies. Lakes are the key link of the surface water facies shifting and water cycling on the Tibetan Plateau. Lake areas are sensitive to precipitation changes of the westerlies and the Indian

* 2020-02-15 收稿; 2020-03-21 收修改稿.

国家第二次青藏高原综合科学考察研究项目 (2019QZKK0202)、国家自然科学基金重点项目 (41831177)、中国科 学院战略性先导科技专项项目 (A 类) (XDA20020102) 和中国科学院野外站联盟项目 (KFJ-SW-YW038) 联合资助.

** 通信作者;E-mail:lpzhu@ itpcas.ac.cn. 
monsoon dominant areas, but the responses of lake area and lake water storage are not always consistent in different regions and period. Lake water temperature has obvious response to climate warming, and the changes of lake water temperature and thermocline depth have obvious influence on the heat exchange of water-atmosphere, thus affecting the regional water cycle processes such as evaporation and precipitation. Due to the increase of lake water storage, the salinity of lakes in the Serling Co area has generally decreased since the 1970s. Based on the remote sensing inversion model constructed by the in-situ investigation of more than 60 lakes, it is found that the lake transparency has generally increased during 2000 and 2019. In terms of water balance monitoring in the big lakes of different supply types, the meteorological and hydrological factors affecting the lake change are quite different. In the current warm and humid climate state, lakes of the Tibet Plateau will continue to expand. In order to further understand the role of lake changes in the regional water cycle and climate change of the Tibetan Plateau, it is necessary to fully understand the variation of lake water storage and their continuous time series, to deeply understand the changes of lake physical and chemical parameters and the effect on heat exchange between lakes and atmosphere, and to obtain more comprehensive and continuous observation data from the great lake basins.

Keywords: Tibetan Plateau; lake area and water storage; water balance; physical and chemical parameters; investigation and observation

青藏高原是地球上最重要的高海拔地区之一,对全球变化具有敏感的响应. 湖泊是大气圈、水圈和冰冻 圈联系的关键纽带,在全球变暖条件下对地表水循环过程具有重要的影响. 已有的观测研究发现, 19602012 年间, 青藏高原地区的气温上升率约为 $0.3 \sim 0.4^{\circ} \mathrm{C} / 10 \mathrm{a}$, 是全球平均水平的 2 倍 ${ }^{[1]}$. 青藏高原的降水变 化区域差异显著. 受西风影响的中部和北部降水自 1990 年代中期以来增加约 $21 \% \pm 7 \%^{[2]}$, 而受季风影响的 高原南部和东部降水减少 ${ }^{[3-4]}$, 呈现波动性下降 ${ }^{[5]}$. 总的来讲, 青藏高原的降水在 2000-2018 年呈现出持续 增加, 水循环总体加剧 ${ }^{[6-7]}$.

在急剧升温的背景下, 与全球平均水平相比, 喜马拉雅山地区的冰川正在经历快速退缩. 喜马拉雅一喀 喇昆仑一帕米尔地区的冰川融水作为 “亚洲水塔” 的重要水源, 对青藏高原及周边地区人类社会发展起着重 要的支撑作用 ${ }^{[8]}$. 随着气温和降水的变化, 青藏高原地区的冰川退缩也表现出明显的区域分异, 具有南部和 东南部冰川退缩强烈、中部冰川退缩程度减弱和西北部冰川稳定甚至前进的特点 ${ }^{[4]}$. 气温升高使得多年冻 土发生明显退化, 1980- 2015 年期间,青藏公路沿线多年冻土活动层底部温度上升速率为 $0.45^{\circ} \mathrm{C} / 10 \mathrm{a}$, 活动 层厚度变化速率为 $21.7 \mathrm{~cm} / 10 \mathrm{a}$. 退化的冻土较之前释放了更多的水分, 1980-2018 年 6-8 月多年冻土区 土壤平均含水量递增率为 $0.005 \mathrm{~m}^{3} /\left(\mathrm{m}^{3} \cdot 10 \mathrm{a}\right)^{[9]}$.

降水增加、冰川融水和冻土融化极大改变了青藏高原的湖泊面积和水量. 根据对青藏高原中西部面积 大于 $10 \mathrm{~km}^{2}$ 的湖泊水量变化的分析, 1976-1990 年,湖泊水量因降水量减少而下降; 1990-2013 年, 又因降 水量和冰川融水增加而上升, 其中, 冰川补给湖泊的水量增加占研究地区和时段全部湖泊水量增加的 $84 \%^{[10]}$. 湖泊面积大小和水量变化显然会影响水分向大气的输送. 研究发现, 对纳木错流域的开阔湖区和岸 边小湖的对比研究发现, 开阔湖区的热量和水汽总体传输系数比岸边小湖低 7\%左右, 而非结冰期的湖面蒸 发比小湖要高 ${ }^{[11]}$. 由于湖泊减少了陆地表面向大气输送的能量, 大面积的湖泊扩张反过来也会促使区域降 水增加 ${ }^{[12]}$.

青藏高原的湖泊分布广泛而密集. 遥感反演结果表明,2018 年湖泊总面积约为 $5 \times 10^{4} \mathrm{~km}^{2[13]}$ (图 1 ), 分 别占全球湖泊面积的 $1.9 \%{ }^{[14]}$, 占中国湖泊面积的 $57.2 \%^{[15-16]}$, 占青藏高原陆地面积的 $1.9 \%$. 青藏高原的湖 泊在 “亚洲水塔” 的区域水相转换和水循环中具有十分重要的影响. 在巴黎会议预测未来全球平均气温上升 $2^{\circ} \mathrm{C}$ 的情景下, 青藏高原的气候环境将会发生更加剧烈的变化,迫切需要对青藏高原湖泊变化的幅度、趋势 及对区域气候变化的响应和影响方式与机制开展深人研究. 因此, 国家启动的 “第二次青藏高原科学考察研 究” 专项将开展 “湖泊演变及气候变化响应” 的专题研究, 其中的重要目标就是准确认识青藏高原湖泊水量 赋存形式、类型及转化过程,揭示湖泊水循环及流域生态系统变化对气候变化的响应和和反馈. 


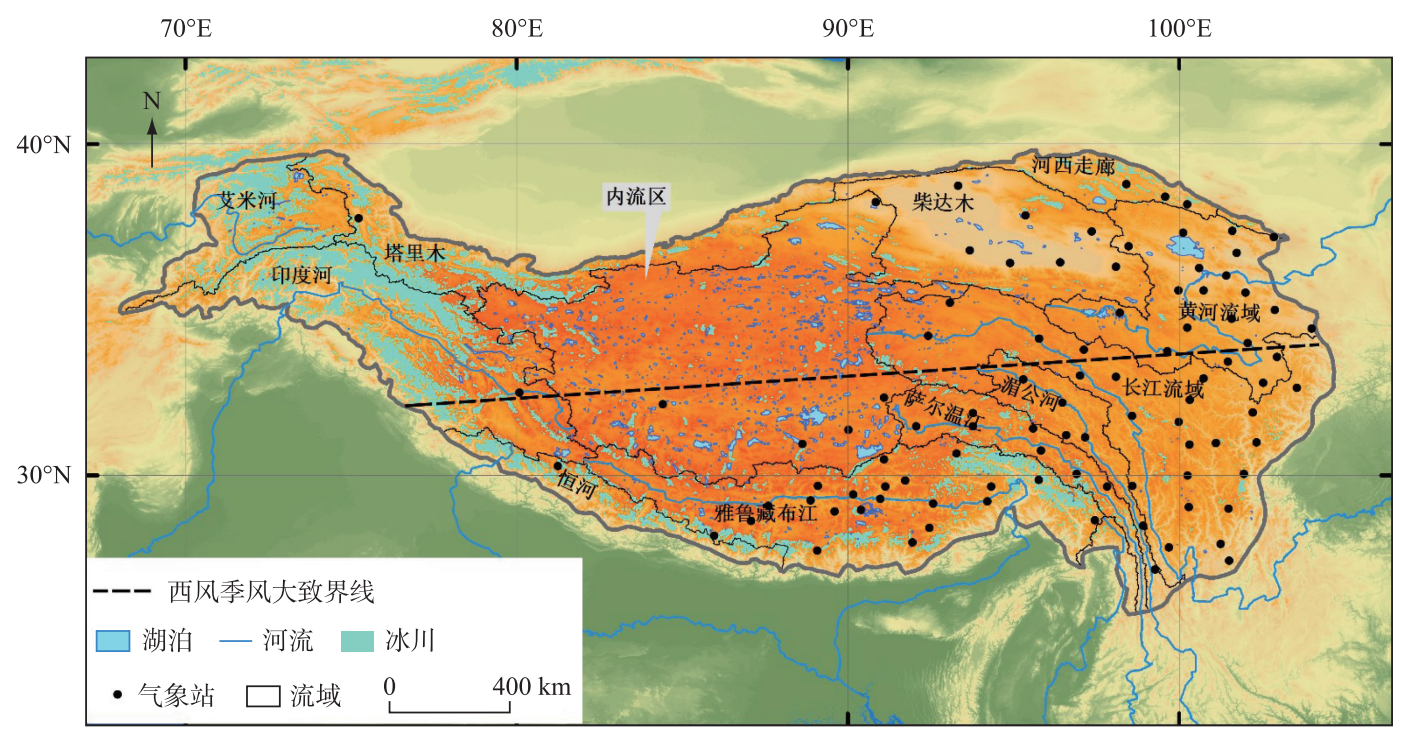

图 1 青藏高原冰川、湖泊和主要河流分布(根据文献[13]修改)

Fig.1 Distribution of glaciers, lakes and major rivers on the Tibetan Plateau (Modified from reference [13])

\section{1 湖泊面积、水位与水量变化}

\section{1 湖泊面积与水位变化}

最近几十年,气候变化对青藏高原的湖泊面积变化具有明显的影响. 对青藏高原不同大小的湖泊面积 变化研究表明,1990 年之前,小于 $10 \mathrm{~km}^{2}$ 的湖泊面积出现微弱扩张,但大于 $10 \mathrm{~km}^{2}$ 的湖泊(占全部湖泊面积 的 70\% 以上) 则呈现普遍退缩. 1990 年之后, 大多数湖泊均呈现扩张态势, 并且在 2000 年之后表现出扩张速 率的增长 ${ }^{[17]}$. 与气象参数进行对比发现, $1970 \mathrm{~s}$ 湖泊萎缩的主要原因是较少的降水和低温对融水释放的限 制, 而 1990s 以来的湖泊扩张与降水和融水增加有关 ${ }^{[18]}$. 一些湖泊的水位实地监测与湖泊面积变化具有相 同的趋势 ${ }^{[19]}$, 其结果也得到基于 ICESat/ICESat-2 和 CryoSat 等遥感监测的大尺度湖泊水位时空变化的 支持 ${ }^{[20-21]}$.

\section{2 湖泊水量变化}

然而,与湖泊面积有大致相似变化的同时,湖泊水量在不同的区域和时间段呈现不同的变化. 研究者利 用重力卫星对水体质量的估算, 结合区域降水量、冰川融化量、土壤含水量等分析了湖泊水量的变化以及各 个补给要素的影响 ${ }^{[22]}$. 以湖盆 DEM 为基础, 建立湖泊面积与水量变化量的关系,则能够估算每个湖泊在面 积变化后的水量变化 ${ }^{[23]}$. 1976-1990 年, 高原内流区东南部湖泊水量增加,而东北部湖泊水量减少, 并明显 快于其他地区. 1990-2000 年,高原内流区东北部水量依然减少,其他地区湖泊水量均增加. 2000-2013 年, 高原内流区大多数湖泊水量增加, 且东部增加明显快于西部, 但藏南地区湖泊水量呈现持续减少 ${ }^{[23]}$ (图 2). 对面积大于 $10 \mathrm{~km}^{2}$ 的 315 个湖泊的水量变化分析表明, 1976-1990 年湖泊水量减少 236.9 亿 $\mathrm{m}^{3}, 1990-$ 2013 年湖泊水量增加 1408 亿 $\mathrm{m}^{3}$,增加的区域主要在高原北部和中部. 2000-2013 年期间, 高原北部和中部 的湖泊面积分别增加了 1981.6 和 $1869.1 \mathrm{~km}^{2}$, 具有几乎一致的面积变化, 但前者的湖泊水量增加只占后者 的一半, 表明湖泊面积的变化幅度可能不能准确反映其对气候变化的响应. 对于整个高原来讲, 降水增加是 2000-2013 年高原湖泊水量增加的主要原因, 但在其北部、西部和西北部地区, 冰川融水湖泊分别占到水量 增加湖泊的 $40 \% 、 50.6 \%$ 和 $100 \%{ }^{[24]}$. 大气环流和降水变化的区域差异表明,青藏高原湖泊水量变化的区域 差异 ${ }^{[25]}$ 与降水变化密切相关. 研究发现, 青藏高原北部的湖泊扩张和南部的湖泊退缩与北部西风区的降水 增加和南部季风区的降水减少具有时间和空间上的一致性 ${ }^{[4]}$. 

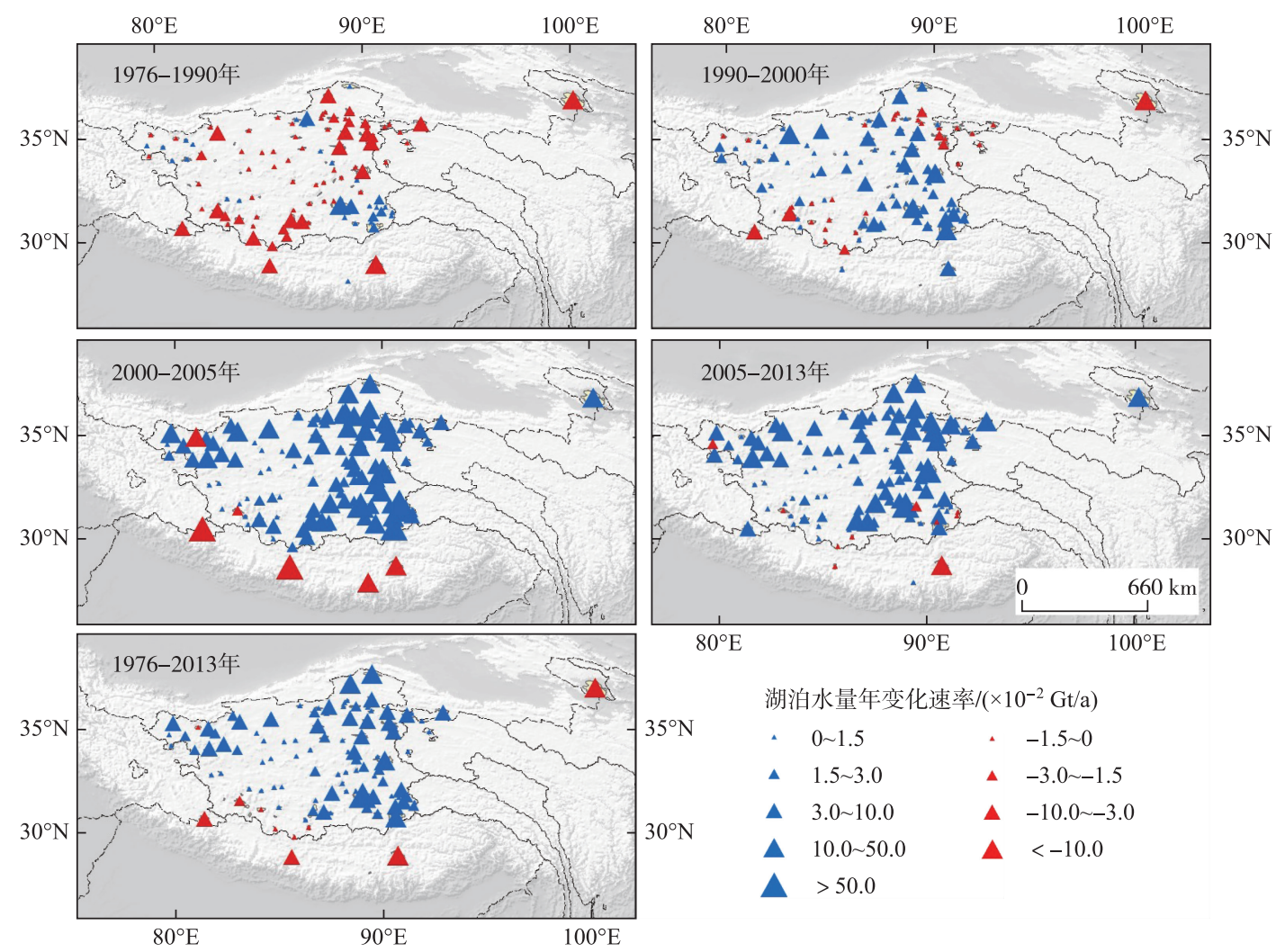

湖泊水量年变化速率 $/\left(\times 10^{-2} \mathrm{Gt} / \mathrm{a}\right)$

. $0 \sim 1.5$. $-1.5 \sim 0$

$\triangle \quad 1.5 \sim 3.0 \quad \Delta \quad-3.0 \sim-1.5$

$\Delta \quad 3.0 \sim 10.0 \quad \Delta \quad-10.0 \sim-3.0$

$\Delta \quad 10.0 \sim 50.0 \quad \boldsymbol{\Delta}<-10.0$

$\Delta>50.0$

图 2 青藏高原面积大于 $50 \mathrm{~km}^{2}$ 的封闭湖泊在 1976-1990、1990-2000、2000-2005、 2005-2013 和 1976-2013 年 5 个时段的年均水量变化量 (根据文献[23] 修改)

Fig. 2 The average annual water storage variations of the lakes with areas great than $50 \mathrm{~km}^{2}$ on the Tibetan Plateau during the five periods of 1976-1990, 1990-2000, 2000-2005, 2005-2013 and 1976-2013 (Modified from reference [23])

\section{2 湖泊物理与化学指标变化}

\section{1 湖泊水温与冰情}

青藏高原的湖泊水温对气候变化具有明显的响应. 根据 MODIS/Terra 8 的陆表日温 (夜间) 产品 (MOD11A2) 分析, 2001-2012 年期间,青藏高原湖泊表面水温平均上升率为 $0.012 \pm 0.033^{\circ} \mathrm{C} / \mathrm{a}^{[26]}$. 在调查的 52 个湖泊中, 31 个湖泊 (占 $60 \%$ ) 的平均升温率为 $0.055 \pm 0.033^{\circ} \mathrm{C} / \mathrm{a}$, 其余位于高海拔范围 (> $4200 \mathrm{~m}$ ) 的 21 个湖泊 (占 $40 \%$ ) 的平均降温率为 $-0.053 \pm 0.038^{\circ} \mathrm{C} / \mathrm{a}$ ( 图 3). 湖泊表面水温差异和变化可能与湖泊海拔和冰 雪融水补给程度有关. 例如, 非冰川补给湖泊的平均升温率为 $0.038^{\circ} \mathrm{C} / \mathrm{a}$, 远高于冰川补给湖泊的 $0.004^{\circ} \mathrm{C} / \mathrm{a}$. 湖泊水面温度变化与湖泊冰期具有较高的负相关 ${ }^{[26-27]}$, 表明湖泊冰期缩短可以使空气温度更长时期加热湖 泊水面,导致湖泊加速变暖.

湖冰物候的变化同样反映了气候变暖对湖泊表层水温的影响. MODIS 的冰雪产品显示, 青藏高原的湖 泊普遍在 10 月下旬开始冻结, 在 3 月下旬开始解冻, 湖泊的冰封期在 1 月中旬开始, 在 5 月初结束. 根据对 拥有湖冰数据的 58 个湖泊分析, 其中 40 个湖泊在 2000- 2017 年的冰封持续时间以 $0.8 \mathrm{~d} / \mathrm{a}$ 的速度缩短, 但 也有 18 个湖泊冰封持续时间以 $1.11 \mathrm{~d} / \mathrm{a}$ 的速度增加 ${ }^{[28]}$. 高原中一北部与中一南部的湖泊相比, 前者的结冰 时间较早, 融化时间较晚 ${ }^{[28-29]}$.

湖泊水温及其垂直剖面的季节变化对气温变化具有很好地响应. 对纳木错 2012-2014 年近 3 年的连 


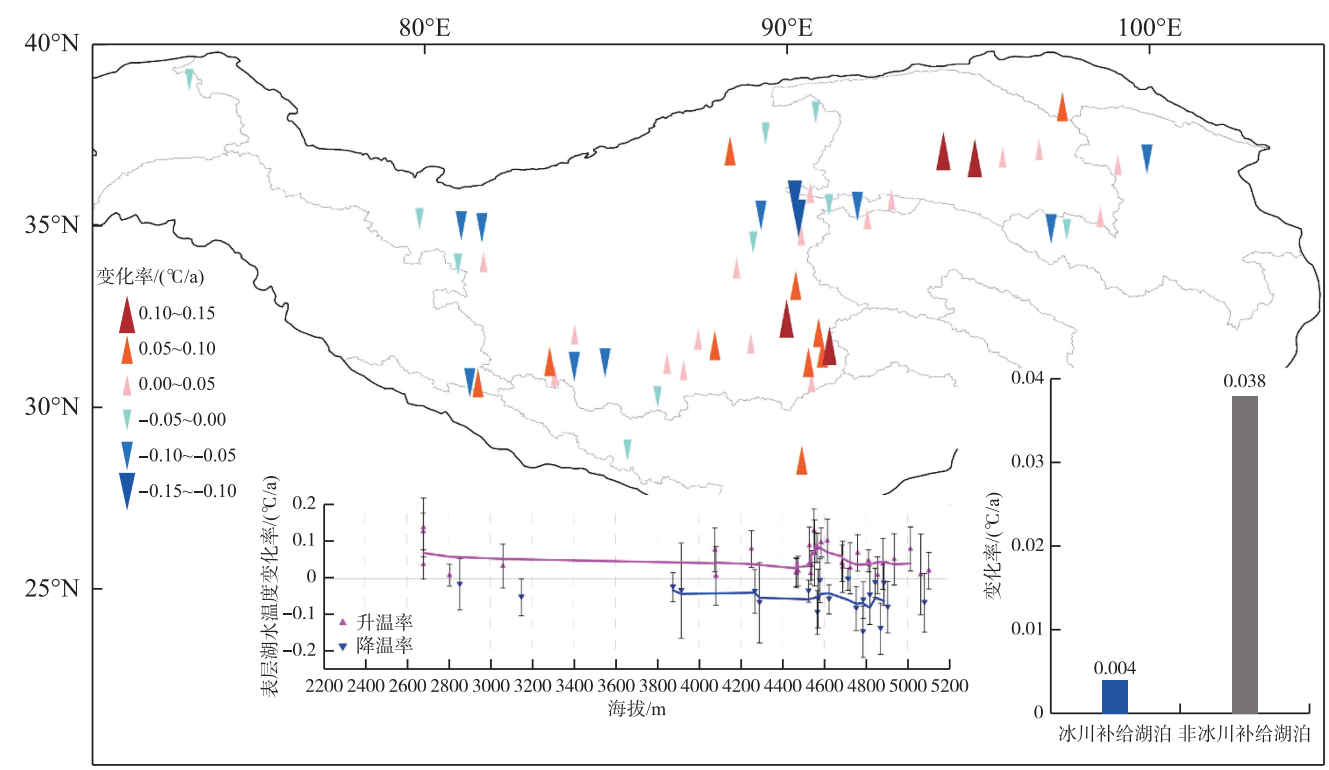

图 3 青藏高原不同类型和海拔湖泊水面温度及其变化速率 (根据文献[26]修改)

Fig.3 Water surface temperature (WST) of the lakes with different types and elevations on the Tibetan Plateau (Modified from reference [26])

续垂直剖面水温观测发现, 湖泊水温剖面变化显示为春季混合一夏季分层一秋季混合一冬季冰封, 具有双对 流 (dimictic) 湖泊的特点, 而在秋季混合期湖泊水温最低降到 $0.5^{\circ} \mathrm{C}$, 远低于其最大密度的温度 $\left(\right.$ 约 $3.6^{\circ} \mathrm{C}$ ). 尽管表层水温与气温 (日均温) 有显著对应关系, 但其变化滞后 (38 d), 显示了湖水对热量传递的调节作 用 ${ }^{[30]}$. 基于气温与湖泊表层水温以及湖水稳定度的关系, 可利用气温预测湖水水温以及热力学稳定度的变 化. 对纳木错水温与气温变化的研究发现, $1979-2012$ 年夏季表层湖水温度平均升高率为 $0.52 \pm 0.25^{\circ} \mathrm{C} /$ $10 \mathrm{a}$, 湖水温跃层的分层开始日期以 $4.20 \pm 2.02 \mathrm{~d} / 10 \mathrm{a}$ 的速率提前, 而分层的持续时间以 $6.00 \pm 3.54 \mathrm{~d} / 10 \mathrm{a}$ 的速率递增 ${ }^{[31]}$. 气温与湖水温度的变化同样改变着湖水的蒸发, 利用纳木错 2011 年 11 月- 2014 年 7 月不 同深度的日平均水温变化数据, 对湖泊一大气热交换的一维模型进行了校正, 发现纳木错平均年蒸发量约为 $832 \pm 69 \mathrm{~mm}$, 比使用 Penman-Monteith 方程估计的潜在蒸发量要小得多. 自 1990s 末以来, 气温升高使得湖泊 平均蒸发量比以前更大,蒸发的增强可能对纳木错的最近扩张起到一定抑制作用 ${ }^{[32]}$.

\section{2 湖泊透明度与盐度}

湖泊透明度的变化主要受湖水中光学组分的影响,包括浮游生物、悬浮颗粒物、有色可溶性有机物 ( CDOM) 以及纯水本身等 ${ }^{[33]}$. 基于青藏高原地区 24 个湖泊实测透明度 ( 以 Secchi Depth 为指标, 简称 SD 值) 和相应的 MODIS 遥感影像湖泊反射率, 建立了该地区湖泊水体 SD 值 MODIS 遥感影像反射率的反演模 型. 结果表明, 基于 MODIS 绿色波段 B4 的单波段幂函数模型在该地区反演效果最好 ${ }^{[34]}$. 进一步分析显示, 青藏高原面积大于 $50 \mathrm{~km}^{2}$ 的湖泊 SD 值在 2000-2019 年平均增加了 $0.0338 \mathrm{~m} / \mathrm{a}(P<0.01)$ (图 4). 湖泊 SD 值的季节性波动幅度为 $1 \sim 2 \mathrm{~m}$. 湖泊 SD 值的变化与降水率密切相关, 也与湖水盐度具有密切联系.

对于青藏高原地区的大多数封闭湖泊来讲, 进人湖泊的水化学离子在水中达到一定的溶解一沉淀平衡, 进而决定着湖泊的盐度 ${ }^{[35]}$. 气候变化在影响湖泊水量平衡的过程中, 降水、融水等淡水补给以及强烈蒸发 等水量损耗, 均对进入湖水的水化学离子含量产生影响, 特别是一些特征离子总是趋于在新的水量平衡条 件下达到新的溶解一沉淀平衡, 从而改变了湖水的盐度. 根据 2017 年对色林错地区的湖泊考察, 将其与 1976 年的考察结果进行比较 (表 1), 可见色林错和达则错的矿化度都降低了 $1 / 3$ 左右, 而赛布错降低高达 $71 \%$, 然而也有一些湖泊 (如达如错) 的矿化度出现增加. 湖泊矿化度变化受多种因素影响, 如气候变化的多个方 面 (降水、蒸发、温度等)、冰融水补给、冻土融化等等, 而且也受湖泊本身的特性控制, 如湖泊面积、水量大 


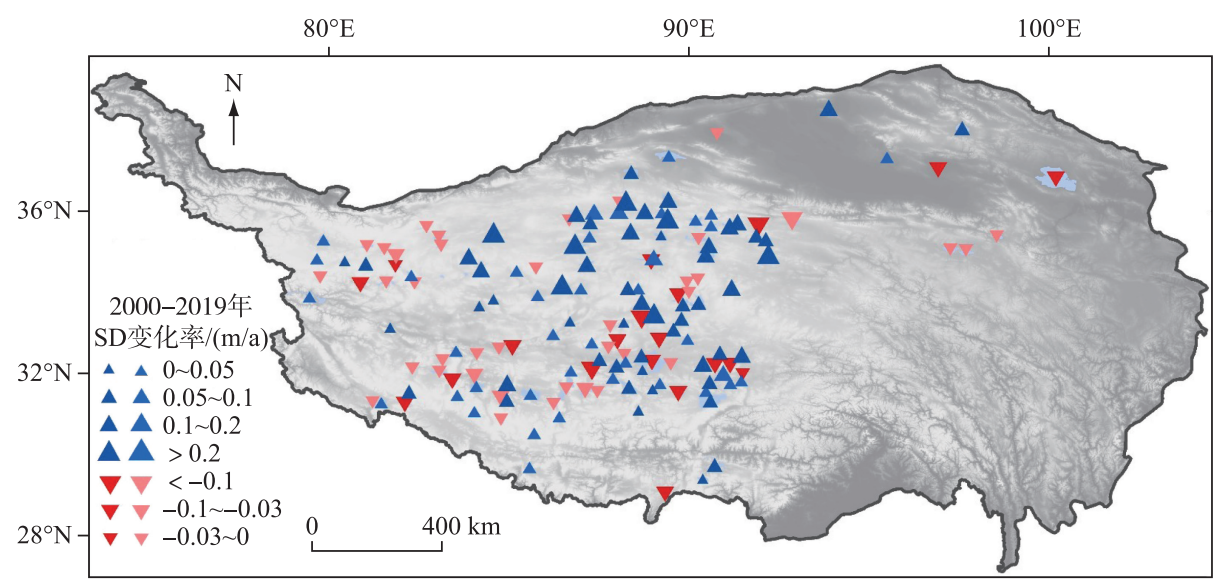

图 4 青藏高原面积大于 $50 \mathrm{~km}^{2}$ 的湖泊 2000-2019 年透明度 SD 值的变化

(三角形大小对应于变化率(线性回归的斜率). 正三角形 (94 个) 表示 SD 增加, 倒三角形 ( 58 个) 表示 SD 减少. 深蓝色正三角形 (54 个) 和暗红色倒三角形 (22 个) 代表通过了显著性 $T$ 检验 $(P<0.05)$, 浅蓝色正三角形和粉红色倒三角形表示未通过 $T$ 检验 $(P>0.05))$

Fig.4 Variations of water transparency (SD) of lakes with area greater than $50 \mathrm{~km}^{2}$ on the Tibetan Plateau between 2000 and 2019 ( The size of triangles corresponds to variation values (slope of linear regression). Upward triangles ( 94 sites) indicate SD increasing while downward triangles ( 58 sites) indicate SD decreasing.

Deep blue ( 54 sites) and dark red ( 22 sites) triangles mean passing a significance $T$-test $(P<0.05)$, while light blue and peach triangles indicate they did not pass the $T$-test $(P>0.05))$

小、盐度水平等, 但湖泊矿化度的普遍下降应与近年大量淡水注人造成的湖泊扩张有关 ${ }^{[36]}$.

表 1 青藏高原色林错地区部分湖泊水质参数 40 年前后的数据对比

Tab.1 Comparison of water quality parameters of some lakes in the Serling Co area on the Tibetan Plateau

\begin{tabular}{|c|c|c|c|c|c|c|}
\hline \multirow{2}{*}{ 湖泊名称 } & \multicolumn{2}{|c|}{ 矿化度/(g/L) } & \multicolumn{2}{|c|}{ 透明度/m } & \multicolumn{2}{|c|}{$\mathrm{pH}$} \\
\hline & 1976 年 & 2017 年 & 1976 年 & 2017 年 & 1976 年 & 2017 年 \\
\hline 色林错 & 18.54 & 12.45 & $7.5 \sim 8.0$ & $3.0 \sim 3.5$ & 9.55 & 9.6 \\
\hline 错鄂 & 0.516 & 0.434 & 11.5 & 8.0 & 7.0 & 9.52 \\
\hline 格仁错 & 0.261 & 0.263 & - & $6.0 \sim 6.7$ & 9.9 & - \\
\hline 达则错 & 40.61 & 27.69 & 7.2 & $4 \sim 7$ & 9.6 & 10.1 \\
\hline 赛布错 & 28.35 & 8.29 & - & - & 9.6 & 9.65 \\
\hline 尔阿错 & - & 4.43 & - & 2.8 & - & 9.68 \\
\hline 果根错 & 1.99 & 11.14 & - & - & - & 9.87 \\
\hline 瀑赛尔错 & 12.45 & 13.95 & - & 2 & - & 9.78 \\
\hline 兹格塘错 & 35.90 & 29.91 & 6.7 & 3.25 & 10.1 & 10 \\
\hline 达如错 & 6.93 & 11.41 & - & 0.94 & 8.3 & 9.3 \\
\hline 江错 & 25.67 & 31.92 & - & 2 & 9.5 & 9.15 \\
\hline 巴木错 & 16.66 & 7.51 & - & 1.9 & 9.6 & 9.7 \\
\hline
\end{tabular}

\section{3 典型湖泊的水量平衡及气候变化响应}

\section{1 纳木错水量平衡研究}

纳木错目前是青藏高原第 3 大湖泊, 流域面积 $10610 \mathrm{~km}^{2}$, 冰川覆盖率约为 $1.7 \%$, 冰川沿念青唐古拉山 分布并靠近陡峭的湖岸, 融水大部分直接人湖. 利用航测地形图、遥感影像数据、实测水深数据以及流域地 
区的气象资料,对纳木错的水量平衡分析表明,1971-2004 年期间, 湖泊水量从 783.23 亿 $\mathrm{m}^{3}$ 增加到 863.77 亿 $\mathrm{m}^{3}$, 平均增加速率为 2.37 亿 $\mathrm{m}^{3} / \mathrm{a}$. 其中, 湖泊水量在 1992-2004 年的增加速率为 3.61 亿 $\mathrm{m}^{3} / \mathrm{a}$, 高于其在 1971-1991 年的增加速率 (1.60 亿 $\left.\mathrm{m}^{3} / \mathrm{a}\right)$. 两个研究时段内, 湖面降水与陆面降水产生的径流补给分别占 湖泊总补给量的 $63.0 \%$ 和 $61.9 \%$, 而冰川融水补给仅占总补给量的 $8.6 \%$ 和 $11.5 \%$, 显示降水是构成湖泊补 给的主要来源. 然而, 从湖泊水量增加的原因来分析, 降水增加及其产生的径流对湖泊总补给增量的贡献率 为 $46.7 \%$, 而冰川融水增加对湖泊总补给增量的贡献率则高达 $52.9 \%$, 显示气候变暖引起的冰川融水增加 是引起近年纳木错湖面迅速扩张的主要原因 (表 2$)^{[37-38]}$.

表 2 纳木错水量平衡各要素及其变化情况 $(A=B+C$,引自文献[37] $)$

Tab.2 The factors and their variations of water balance of Nam Co drainage basin $(\mathrm{A}=\mathrm{B}+\mathrm{C}$, from reference $[37])$

\begin{tabular}{|c|c|c|c|c|c|c|c|}
\hline \multirow{2}{*}{\multicolumn{2}{|c|}{ 水量平衡要素 }} & \multicolumn{2}{|c|}{$1971-1991$ 年 } & \multicolumn{2}{|c|}{$1992-2004$ 年 } & \multicolumn{2}{|c|}{$\begin{array}{c}1971-1991 \text { 年与 } \\
1992-2004 \text { 年比较 }\end{array}$} \\
\hline & & $\begin{array}{l}\text { 年均值/ } \\
\left(\text { 亿 } \mathrm{m}^{3} / \mathrm{a}\right)\end{array}$ & $\begin{array}{l}\text { 占总补给 } \\
\text { 比例/\% }\end{array}$ & $\begin{array}{l}\text { 年均值/ } \\
\left(\text { 亿 } \mathrm{m}^{3} / \mathrm{a}\right)\end{array}$ & $\begin{array}{l}\text { 占总补给 } \\
\text { 比例/\% }\end{array}$ & $\begin{array}{c}\text { 年均值变化/ } \\
\left(\text { 亿 } \mathrm{m}^{3} / \mathrm{a}\right)\end{array}$ & $\begin{array}{c}\text { 占年均值 } \\
\text { 变化比例 } / \%\end{array}$ \\
\hline \multirow[t]{5}{*}{ 总补给 $(A)$} & 湖面降水(A1) & 8.07 & 27.2 & 8.47 & 26.6 & 0.40 & 19.1 \\
\hline & 陆面降水径流( A2) & 10.64 & 35.8 & 11.22 & 35.3 & 0.58 & 27.6 \\
\hline & 冰川融水 (A3) & 2.54 & 8.6 & 3.65 & 11.5 & 1.11 & 52.8 \\
\hline & 其他补给 ( A4) & 8.45 & 28.5 & 8.46 & 26.6 & 0.01 & 0.5 \\
\hline & 补给总量 (A) & 29.70 & 100 & 31.80 & 100 & 2.10 & 100 \\
\hline \multirow{2}{*}{$\begin{array}{c}\text { 总消耗与水量 } \\
\text { 变化 }(B+C)\end{array}$} & 湖面蒸发 (B) & 28.10 & 94.6 & 28.19 & 88.7 & 0.09 & 4.3 \\
\hline & 湖泊水量变化 $(\mathrm{C})$ & 1.60 & 5.4 & 3.61 & 11.3 & 2.01 & 95.7 \\
\hline
\end{tabular}

\section{2 色林错水量平衡研究}

色林错近 10 年来扩张明显, 已经由青藏高原第 3 大湖上升为第 2 大湖, 流域面积 $45530 \mathrm{~km}^{2}$, 冰川覆盖 率约为 $0.6 \%$, 由于流域内的冰川面积相对比例较小, 并且都在远离湖泊的流域边界, 融水只能通过较长距离 的河流补给人湖, 而在高原北部强烈的蒸发作用下, 冰川融水沿途损耗较大. 利用色林错湖面蒸发模拟结 果 ${ }^{[39]}$, 结合中国区域地面气象要素数据集 (CMFD) 的湖面降水数据 ${ }^{[00]}$ 和径流模拟结果 ${ }^{[41]}$, 表 3 给出了 1979-2013 年色林错湖泊水量平衡各要素的统计结果. 可以看出, 1979-1999 年和 2000-2013 年两个时段 内湖面降水、非冰川径流、冰川融水对色林错湖泊水量的补给比例分别为 $24.6 \%$ 、68.0\%、7.4\% 和 $18.5 \%$ 、 $74.7 \% 、 6.8 \%$, 因此, 降水以及降水相关的陆面径流是色林错湖泊补给的主要成分, 占到 $90 \%$ 以上, 而冰川融 水对色林错湖泊水量补给只占 7\%左右. 1979-1999 年期间补给到色林错的水量有 94.3\% 以蒸发的形式消 耗掉, 剩余 5.7\% 水量留在湖泊内; 而 2000-2013 年时段内补给到色林错的水量仅有 $54.4 \%$ 以蒸发形式损 耗, 而 $45.6 \%$ 的水量留在湖泊内 ${ }^{[42]}$.

表 3 色林错湖泊流域水量平衡各要素统计 (引自文献 $[39,42]$ )

Tab.3 Statistics on the factors of water balance in the Serling Co drainage basin (from reference $[39,42]$ )

\begin{tabular}{|c|c|c|c|c|c|c|}
\hline \multirow{2}{*}{ 时期 } & \multirow{2}{*}{ 指标 } & \multicolumn{3}{|c|}{ 年均补给量 } & \multirow{2}{*}{$\begin{array}{c}\text { 年均消耗 } \\
\text { 蒸发 }\end{array}$} & \multirow{2}{*}{ 年均水量变化 } \\
\hline & & 湖面降水 & 非冰川径流 & 冰川径流 & & \\
\hline \multirow[t]{2}{*}{ 1979-1999 年 } & 水量 $/\left(\right.$ 亿 $\left.\mathrm{m}^{3}\right)$ & 5.0 & 13.7 & 1.5 & 19.1 & 1.2 \\
\hline & 比例 $/ \%$ & 24.6 & 68.0 & 7.4 & 94.3 & 5.7 \\
\hline \multirow[t]{2}{*}{$2000-2013$ 年 } & 水量/ $\left(\right.$ 亿 $\left.\mathrm{m}^{3}\right)$ & 7.6 & 30.7 & 2.8 & 22.4 & 18.8 \\
\hline & 比例 $\% \%$ & 18.5 & 74.7 & 6.8 & 54.4 & 45.6 \\
\hline \multirow[t]{2}{*}{$1979-2013$ 年 } & 水量 $/\left(\right.$ 亿 $\left.\mathrm{m}^{3}\right)$ & 6.0 & 20.5 & 2.0 & 20.4 & 8.2 \\
\hline & 比例/\% & 21.1 & 71.8 & 7.1 & 71.3 & 28.7 \\
\hline
\end{tabular}

通过定量分析, 1979-2013 年期间湖面蒸发减小对色林错湖泊扩张的贡献为 $14.0 \%$, 而湖面降水、非冰 
川径流和冰川融水的增加对色林错湖泊扩张的贡献分别为 9.5\%、67.0\% 和 9.5\%. 因此,近 30 年来色林错湖 泊扩张的主导因素是流域内陆面降水产生非冰川径流的增加, 而湖面蒸发的减弱也是促进湖泊扩张的重要 因素 ${ }^{[39,42]}$.

\section{4 湖泊变化对区域环境和气候变化的影响}

\section{1 湖泊对区域气候变化的影响}

青藏高原的湖泊变化不仅对气候变化具有敏感的响应, 也对区域尺度的气候变化具有明显影响, 同时 也可能通过大气环流对更大范围的气候或天气格局产生效应. 在夏季, 大面积的高原湖泊存在能够降低局 地地面空气温度, 并增强中尺度 (湖面和周围地区) 的降水 ${ }^{[43-4]}$. 这些湖泊还通过削弱感热通量和加强潜热 通量来改变区域尺度的大气环流 ${ }^{[4]}$. 在纳木错地区, 模型的敏感性实验发现, 湖泊的季节性冷/热效应导致 流域的降水出现明显的空间差异 ${ }^{[45]}$. 天气研究和预报 (WRF) 模拟显示, 纳木错流域在一些气候极端事件中 (如 2006 年 10 月 24 日的暴风雪), 下风向的降水显著增加 (高达 70\% ) ${ }^{[45]}$. 纳木错湖泊的这种冷却效应甚 至推迟了对流边界层水热交换时间 ${ }^{[46]}$.

\section{2 湖泊对大气环流的影响}

一般而言, 青藏高原湖泊扩张可以通过增加地表水的覆盖面积来影响蒸发. 然而, 由于全球陆地蒸散 (ET) 产品的空间分辨率限制 (1 km 或更大) 以及对水面蒸发重建结果的较大不确定性 ${ }^{[47-48]}$, 目前的数据还 难以评估单个高原湖泊面积扩展/收缩引起的蒸发变化. 一些研究表明, 因为高原湖泊普遍具有较高的盐度 和 $\mathrm{pH}$ 值, 水温变化可能在湖泊沿岸带释放更多的温室气体 $\left(\mathrm{CO}_{2} 、 \mathrm{CH}_{4} 、 \mathrm{~N}_{2} \mathrm{O}\right)^{[49]}$. 因而, 需要开展更广泛的观 测和研究,才能多方面理解湖泊扩张对区域气候变化和生态系统的反馈.

\section{5 湖泊变化对未来气候变化的响应}

\section{1 基于水量平衡的湖泊未来变化}

随着气候持续变暖, 青藏高原未来的湖泊将会持续出现明显的改变. 青藏高原的湖泊变化受降水、蒸发 以及气温增加引起的冰川融水变化影响, 而湖泊蒸发又与表层水温和风速具有密切联系 ${ }^{[39]}$. 对纳木错的表 层水温监测与模拟表明, 湖泊表层水温随着气温升高和长波辐射增加呈现明显的增加趋势 ${ }^{[31]}$. 通过综合考 虑上述要素建立的湖泊水量平衡模型, 反推了引起湖泊水量变化的气象要素变化 ${ }^{[2]}$. 结果表明, 根据高原内 陆封闭湖泊面积在 1995-2015 年由 $24930 \mathrm{~km}^{2}$ 增加到 $33741 \mathrm{~km}^{2}$ (增幅达 $35 \%$ ) 的事实 ${ }^{[22]}$, 反推的降水增幅 达 $21 \% \pm 7 \%$, 反推结果与“全球降水气候中心” (Global Precipitation Climatology Centre,GPCC) 的同化结果 ${ }^{[50]}$ 具有高度一致性 (图 5). 利用该模型预测,2015-2035 年高原内陆封闭湖泊面积将持续扩大,其中前 10 年 (2016-2025 年) 在气候变化速率可能与现在近似的情况下, 湖泊面积将继续增加 $4000 \mathrm{~km}^{2}$, 但速率低于其
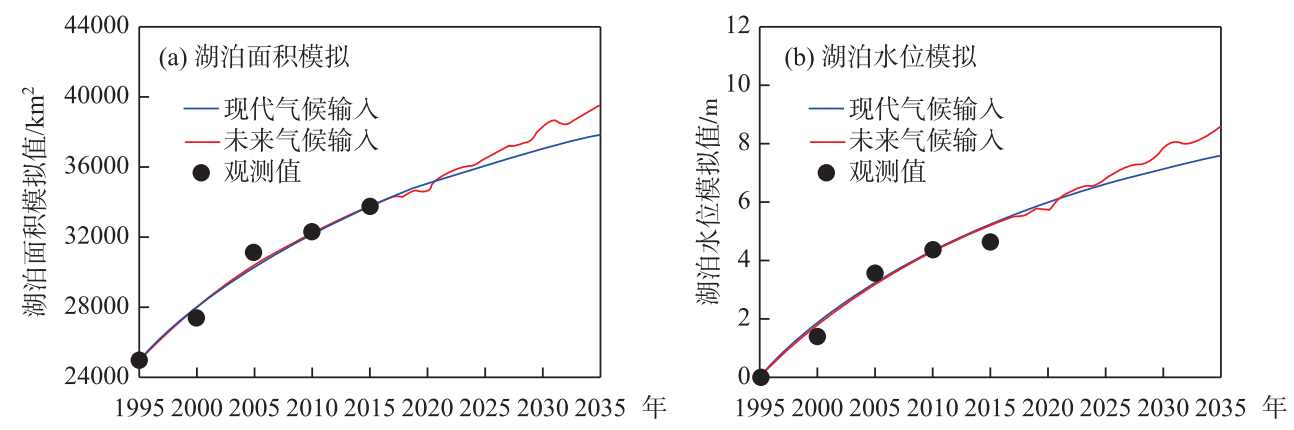

图 5 在当前气候变化状态和利用 Budyko(1958) ${ }^{[51]}$ 关系和蒸发不变假设的未来气候变化驱动下, 对湖泊面积和水位的平均增长进行预测的结果(引自文献 [2])

Fig.5 The results of the prediction of the average increase in lake area and water level, driven by the current climatic state and future climate change using the Budyko (1958) ${ }^{[51]}$ relationship and the evaporation-invariant assumption (from reference [2]) 
在 1995-2015 年的扩张速率; 而后 10 年由于气候的温暖湿润程度更加强烈, 湖泊可能出现更强的扩张. 然 而, 这种预测仅通过量化未来的降水来实现, 而较少考虑湖泊蒸发和冰川融化的影响, 其结果仍然具有较大 的不确定性 ${ }^{[2]}$.

\section{2 基于气候预测的湖泊未来响应}

通过气候变化模型预测显示, 与 1961-1990 年相比, 2015-2050 年高原内陆湖区降水量将增加 $10.4 \% \sim 11 \%{ }^{[1]}$. 在气候变化 RCP4.5 情景下, 到 2030 年冰川将减少 $9 \% \sim 32 \%$, 在 RCP8.5 情境下将减少 $8.7 \% \sim 26.1 \%{ }^{[50-55]}$, 而将降水和冰川变化对湖泊水量平衡的贡献综合考虑, 高原内陆湖泊水量在近期 (约 2030 年) 就会大幅增加 ${ }^{[22]}$. 此外, 由于青藏高原降水受到大西洋年代际振荡 (AMO) 的影响, 未来湖泊面积 变化的预测可能需要考虑多年代际变化和全球变暖的效应.

\section{6 深入认识湖泊变化在区域水循环和气候变化中的作用}

青藏高原的湖泊变化不仅通过不同类型的补给变化反映气候变化及各要素的影响过程,而且由于湖泊 面积、水量和理化性质的改变对水热交换产生明显影响, 从而对气候变化具有一定的反馈. 因此, 在正在启 动的 “第二次青藏高原综合科学考察研究” 专项中, “湖泊演变及气候变化响应”专题的重要任务之一就是 开展更加精细的湖泊变化与气候变化关系研究, 深人理解湖泊变化在区域水循环中的作用, 从而准确评判 未来气候变化条件下的湖泊变化趋势. 主要研究工作将从以下方面展开:

1) 宏观尺度的湖泊水量赋存. 青藏高原的湖泊有多少水量赋存, 气候变化对其赋存条件和程度具有哪 些影响, 是湖泊研究回答其在 “亚洲水塔” 水循环作用的根本问题. 尽管遥感技术与反演方法的发展使得利 用卫星遥感数据已经较为容易和快捷地获取湖泊的面积指标, 但由于湖盆形状、湖泊深度和岸线坡度的差 异, 不同湖泊之间的面积变化幅度与其水量变化幅度也不一致. 需要根据湖盆数字高程模型 (DEM) 对每一 个湖泊建立符合其本身特点的面积和水量关系, 才能通过遥感大数据获取整个高原各个湖泊不同时期的水 量赋存,分析其补给过程的时空变化,进而准确评价整个青藏高原湖泊水量变化与气候变化的关系.

2) 湖泊主要理化性质的系统调查与分析. 青藏高原的湖泊水体质量如何, 气候变化对其有何影响, 是湖 泊研究回答其在 “亚洲水塔” 中作为可利用水资源的核心问题. 湖泊透明度与盐度变化对气候变化具有敏感 响应, 也通过对水温、蒸发的影响对气候变化产生重要的反馈. 遥感影像提供了长时期、大范围的湖泊水色 光谱数据, 由于湖泊水色敏感地反映湖泊透明度变化, 而一些影像的光谱与盐度之间也具有一定的相关性. 因此, 具有利用遥感影像的湖泊水色和光谱数据重建湖泊透明度与盐度变化的基础. 获取较高精度时间序 列的湖泊透明度和盐度的变化, 对于全面理解湖泊的理化性质及对气候变化的响应具有重要意义.

3) 典型湖泊的水量平衡监测与模型模拟. 西风和印度季风是影响青藏高原地区的两大环流系统. 近年 来, 西风和印度季风作用区出现了明显的降水变化, 从而导致不同地区的湖泊变化具有相应的时空分异. 青 藏高原地区的湖泊除了受降水影响外, 还与流域内的冰川融水、冻土退化等具有紧密联系. 青藏高原的湖泊 变化使得这些湖泊与大气间的水分和热量交换发生明显改变,进而影响了区域水循环过程. 然而, 不同地区 影响湖泊水量平衡的主导要素如何, 这些要素在气候变化条件下如何发挥作用, 是从机制上准确理解湖泊 在“亚洲水塔” 的水循环作用的关键. 因而, 在青藏高原各个典型地区加强大湖流域的湖泊水量平衡及其对 气候变化响应研究,开展降水、径流 (包括降水、融水和地下径流)、蒸发等要素的空间与季节分布的观测研 究, 利用水量总平衡和同位素分割等手段, 研究湖泊水量平衡对不同补给来源变化的响应, 能够深人理解气 候变化对湖泊水量变化的影响过程与机制.

\section{7 参考文献}

[ 1 ] Chen DL, Xu BQ, Yao TD et al. Assessment of past, present and future environmental changes on the Tibetan Plateau. Chinese Science Bulletin, 2015, 60(32): 3025-3035. [陈德亮, 徐柏青, 姚檀栋等. 青藏高原环境变化科学评估: 过 去、现在与未来. 科学通报, 2015, 60(32) : 3025-3035.]

[ 2 ] Yang K, Lu H, Yue SY et al. Quantifying recent precipitation change and predicting lake expansion in the Inner Tibetan Plateau. Climatic Change, 2018, 147(1/2) : 149-163. DOI : 10.1007/s10584-017-2127-5.

[ 3 ] Yang K, Ye BS, Zhou DG et al. Response of hydrological cycle to recent climate changes in the Tibetan Plateau. Climatic 
Change, 2011, 109(3/4) : 517-534. DOI: 10.1007/s10584-011-0099-4.

[ 4 ] Yao TD, Thompson L, Yang W et al. Different glacier status with atmospheric circulations in Tibetan Plateau and surroundings. Nature Climate Change, 2012, 2(9) : 663-667. DOI: 10.1038/nclimate1580.

[ 5 ] Lei YB, Yao TD, Yang K et al. An integrated investigation of lake storage and water level changes in the Paiku Co basin, central Himalayas. Journal of Hydrology, 2018, 562 : 599-608. DOI: 10.1016/j.jhydrol.2018.05.040.

[ 6 ] Zhang GQ, Yao TD, Xie HJ et al. Increased mass over the Tibetan Plateau: From lakes or glaciers? Geophysical Research Letters, 2013, 40(10) : 2125-2130. DOI: 10.1002/grl.50462.

[ 7 ] Gao YH, Cuo L, Zhang YX. Changes in moisture flux over the Tibetan Plateau during 1979-2011 and possible mechanisms. Journal of Climate, 2014, 27(5) : 1876-1893. DOI: 10.1175/jcli-d-13-00321.1.

[ 8 ] Pritchard HD. Asia's shrinking glaciers protect large populations from drought stress. Nature, 2019, 569(7758) : 649-654. DOI : $10.1038 / \mathrm{s} 41586-019-1240-1$.

[ 9 ] Zhao L, Hu GJ, Zou DF et al. Permafrost changes and its effects on hydrological processes on Qinghai-Tibet plateau. Bulletin of Chinese Academy of Sciences, 2019, 34(11): 1233-1246. [ 赵林, 胡国杰, 邹德富等. 青藏高原多年冻土变化 对水文过程的影响. 中国科学院院刊, 2019, 34(11): 1233-1246.]

[10] Qiao BJ, Zhu LP. Difference and cause analysis of water storage changes for glacier-fed and non-glacier-fed lakes on the Tibetan Plateau. Science of the Total Environment, 2019, 693: 133399. DOI: 10.1016/j.scitotenv.2019.07.205.

[11] Wang BB, Ma YM, Wang Y et al. Significant differences exist in lake-atmosphere interactions and the evaporation rates of high-elevation small and large lakes. Journal of Hydrology, 2019, 573: 220-234. DOI : 10.1016/j.jhydrol.2019.03.066.

[12] Dai YF, Yao TD, Li XY et al. The impact of lake effects on the temporal and spatial distribution of precipitation in the Nam Co basin, Tibetan Plateau. Quaternary International, 2018, 475: 63-69. DOI: 10.1016/j.quaint.2016.01.075.

[13] Zhang GQ, Luo W, Chen WF et al. A robust but variable lake expansion on the Tibetan Plateau. Science Bulletin, 2019, 64(18) : 1306-1309. DOI: 10.1016/j.scib.2019.07.018.

[14] Messager ML, Lehner B, Grill G et al. Estimating the volume and age of water stored in global lakes using a geo-statistical approach. Nature Communications, 2016, 7: 13603. DOI: 10.1038/ncomms13603.

[15] Ma RH, Duan HT, Hu CM et al. A half-century of changes in China's lakes: Global warming or human influence? Geophysical Research Letters, 2010, 37(24): L24106. DOI: 10.1029/2010gl045514.

[16] Zhang GQ, Yao TD, Chen WF et al. Regional differences of lake evolution across China during 1960s-2015 and its natural and anthropogenic causes. Remote Sensing of Environment, 2019, 221: 386-404. DOI: 10.1016/j.rse.2018.11.038.

[17] Zhang GQ, Yao TD, Xie HJ et al. Lakes' state and abundance across the Tibetan Plateau. Chinese Science Bulletin, 2014, 59(24) : 3010-3021. DOI: 10.1007/s11434-014-0258-x.

[18] Li JL, Sheng YW, Luo JC et al. Remotely sensed mapping of inland lake area changes in the Tibetan Plateau. J Lake Sci, $2011,23(3)$ : 311-320. DOI : 10.18307/2011.0301. [李均力, 盛永伟, 骆剑承等. 青藏高原内陆湖泊变化的遥感制 图. 湖泊科学, $2011,23(3): 311-320$.]

[19] Lei YB, Yao TD, Bird BW et al. Coherent lake growth on the central Tibetan Plateau since the 1970s: Characterization and attribution. Journal of Hydrology, 2013, 483: 61-67. DOI: 10.1016/j.jhydrol.2013.01.003.

[20] Song CQ, Huang B, Ke LH. Inter-annual changes of alpine inland lake water storage on the Tibetan Plateau: Detection and analysis by integrating satellite altimetry and optical imagery. Hydrological Processes, 2014, 28(4) : 2411-2418. DOI: 10.1002/hyp.9798.

[21] Song CQ, Ye QH, Cheng X. Shifts in water-level variation of Namco in the central Tibetan Plateau from ICESat and CryoSat-2 altimetry and station observations. Science Bulletin, 2015, 60(14) : 1287-1297. DOI : 10.1007/s11434-015-0826-8.

[22] Zhang GQ, Yao TD, Shum CK et al. Lake volume and groundwater storage variations in Tibetan Plateau's endorheic basin. Geophysical Research Letters, 2017, 44(11) : 5550-5560. DOI: 10.1002/2017gl073773.

[23] Yang RM, Zhu LP, Wang JB et al. Spatiotemporal variations in volume of closed lakes on the Tibetan Plateau and their climatic responses from 1976 to 2013. Climatic Change, 2017, 140(3/4) : 621-633. DOI: 10.1007/s10584-016-1877-9.

[24] Qiao BJ, Zhu LP, Yang RM. Temporal-spatial differences in lake water storage changes and their links to climate change throughout the Tibetan Plateau. Remote Sensing of Environment, 2019, 222 : 232-243. DOI: 10.1016/j.rse.2018.12.037.

[25] Lei YB, Yang K, Wang B et al. Response of inland lake dynamics over the Tibetan Plateau to climate change. Climatic Change, 2014, 125(2) : 281-290. DOI: 10.1007/s10584-014-1175-3. 
[26] Zhang GQ, Yao TD, Xie HJ et al. Estimating surface temperature changes of lakes in the Tibetan Plateau using MODIS LST data. Journal of Geophysical Research: Atmospheres, 2014, 119(14) : 8552-8567. DOI: 10.1002/2014jd021615.

[27] Kropácek J, Maussion F, Chen F et al. Analysis of ice phenology of lakes on the Tibetan Plateau from MODIS data. The Cryosphere, 2013, 7(1) : 287-301. DOI: 10.5194/tc-7-287-2013.

[28] Cai Y, Ke CQ, Li XG et al. Variations of lake ice phenology on the Tibetan Plateau from 2001 to 2017 based on MODIS data. Journal of Geophysical Research: Atmospheres, 2019, 124(2) : 825-843. DOI: 10.1029/2018jd028993.

[29] Guo LN, Wu YH, Zheng HX et al. Uncertainty and variation of remotely sensed lake ice phenology across the Tibetan Plateau. Remote Sensing, 2018, 10(10) : 1534. DOI: 10.3390/rs10101534.

[30] Wang JB, Huang L, Ju JT et al. Spatial and temporal variations in water temperature in a high-altitude deep dimictic mountain lake ( Nam Co), central Tibetan Plateau. Journal of Great Lakes Research, 2019, 45(2) : 212-223. DOI: 10. 1016/j.jglr.2018.12.005.

[31] Huang L, Wang JB, Zhu LP et al. The warming of large lakes on the Tibetan Plateau: Evidence from a lake model simulation of Nam Co, China, during 1979-2012. Journal of Geophysical Research: Atmospheres, 2017, 122(24) : 13095-13107. DOI: $10.1002 / 2017 \mathrm{jd} 027379$.

[32] Lazhu, Yang K, Wang JB et al. Quantifying evaporation and its decadal change for Lake Nam Co, central Tibetan Plateau. Journal of Geophysical Research: Atmospheres, 2016, 121(13) : 7578-7591. DOI : 10.1002/2015jd024523.

[33] Mancino G, Nolè A, Urbano V et al. Assessing water quality by remote sensing in small lakes: The case study of Monticchio lakes in southern Italy. IForest-Biogeosciences and Forestry, 2009, 2(4) : 154-161. DOI: 10.3832/ifor0507-002.

[34] Liu C, Zhu LP, Wang JB et al. Remote sensing-based estimation of lake water clarity on the Tibetan Plateau. Progress in Geography, 2017, 36(5): 597-609. [刘肿, 朱立平, 王君波等. 基于 MODIS 的青藏高原湖泊透明度遥感反演. 地 理科学进展, 2017, 36(5): 597-609.]

[35] Wang HL, Zheng MP. Preliminary study of the correlation between hydrochemistry and salinity of lakes in the Qinghai-Tibetan Plateau. Acta Geologica Sinica, 2010, 84(10): 1517-1522. [王海雷, 郑绵平. 青藏高原湖泊水化学与盐度的 相关性初步研究. 地质学报, 2010, 84(10): 1517-1522.]

[36] Second Tibetan Plateau Expedition Survey Team ed. Integrated scientific survey report for environment changes in the Serling Co area of the Tibetan Plateau. Beijing: Science Press, 2020: 105-106. [第二次青藏高原综合科学考察研究队. 西藏色林错地区环境变化综合科学考察报告. 北京: 科学出版社, 2020: 105-106.]

[37] Zhu LP, Xie MP, Wu YH. Quantitative analysis of lake area variations and the influence factors from 1971 to 2004 in the Nam Co basin of the Tibetan Plateau. Chinese Science Bulletin, 2010, 55(13) : 1294-1303. DOI: 10.1007/s11434-0100015-8.

[ 38 ] Zhang B, Wu YH, Zhu LP et al. Estimation and trend detection of water storage at Nam Co Lake, central Tibetan Plateau. Journal of Hydrology, 2011, 405(1/2): 161-170. DOI: 10.1016/j.jhydrol.2011.05.018.

[39] Guo YH, Zhang YS, Ma N et al. Long-term changes in evaporation over Siling Co Lake on the Tibetan Plateau and its impact on recent rapid lake expansion. Atmospheric Research, 2019, 216: 141-150. DOI : 10.1016/j.atmosres.2018.10.006.

[40] He J, Yang K, Tang WJ et al. The first high-resolution meteorological forcing dataset for land process studies over China. Scientific Data, 2020, 7 : 25. DOI: 10.1038/s41597-020-0369-y.

[41] Tong K, Su FG, Xu BQ. Quantifying the contribution of glacier meltwater in the expansion of the largest lake in Tibet. Journal of Geophysical Research: Atmospheres, 2016, 121(19) : 11158-11173. DOI : 10.1002/2016jd025424.

[42] Guo YH, Zhang YS, Ma N et al. Quantifying surface energy fluxes and evaporation over a significant expanding endorheic lake in the central Tibetan Plateau. Journal of the Meteorological Society of Japan Ser II, 2016, 94(5) : 453-465. DOI: 10.2151/jmsj.2016-023.

[43] Wu Y, Huang AN, Yang B et al. Numerical study on the climatic effect of the lake clusters over Tibetan Plateau in summer. Climate Dynamics, 2019, 53(9/10) : 5215-5236. DOI : 10.1007/s00382-019-04856-4.

[ 44] Zhu LJ, Jin JM, Liu X et al. Simulations of the impact of lakes on local and regional climate over the Tibetan Plateau. Atmosphere-Ocean, 2018, 56(4) : 230-239. DOI : 10.1080/07055900.2017.1401524.

[ 45 ] Dai YF, Wang L, Yao TD et al. Observed and simulated lake effect precipitation over the Tibetan Plateau: An initial study at Nam Co Lake. Journal of Geophysical Research: Atmospheres, 2018, 123 ( 13 ): 6746-6759. DOI: 10. 1029/2018jd028330. 
[46] Lv YQ, Ma YM, Li MS et al. Numerical simulation of typical atmospheric boundary layer characteristics over Lake Namco region, Tibetan Plateau in summer. Plateau Meteorology, 2008, 27(4) : 733-740. [吕雅琼, 马耀明, 李茂善等. 纳木 错湖夏季典型大气边界层特征的数值模拟. 高原气象, 2008, 27(4) : 733-740.]

[47] Friedrich K, Grossman RL, Huntington J et al. Reservoir evaporation in the western United States: Current science, challenges, and future needs. Bulletin of the American Meteorological Society, 2018, 99(1) : 167-187. DOI: 10.1175/bams-d15-00224.1.

[48] Wang W, Lee X, Xiao W et al. Global lake evaporation accelerated by changes in surface energy allocation in a warmer climate. Nature Geoscience, 2018, 11(6) : 410-414. DOI: 10.1038/s41561-018-0114-8.

[49] Yan FP, Sillanpää M, Kang SC et al. Lakes on the Tibetan Plateau as conduits of greenhouse gases to the atmosphere. Journal of Geophysical Research: Biogeosciences, 2018, 123(7) : 2091-2103. DOI : 10.1029/2017jg004379.

[50] Schneider U, Becker A, Finger P et al. GPCC's new land surface precipitation climatology based on quality-controlled in situ data and its role in quantifying the global water cycle. Theoretical and Applied Climatology, 2014, 115(1/2) : 15-40. DOI : $10.1007 /$ s00704-013-0860-x.

[51] Budyko MI. The heat balance of the earth's surface. National Weather Service, U. S. Department of Commercial, Washington, D.C, 1958: 144-155.

[52] Bolch T, Shea JM, Liu SY et al. Status and change of the cryosphere in the extended Hindu Kush himalaya region. The Hindu Kush Himalaya Assessment. Cham: Springer International Publishing, 2019: 209-255. DOI : 10.1007/978-3-31992288-1_7.

[53] Giesen RH, Oerlemans J. Climate-model induced differences in the 21 st century global and regional glacier contributions to sea-level rise. Climate Dynamics, 2013, 41(11/12) : 3283-3300. DOI: 10.1007/s00382-013-1743-7.

[54] Marzeion B, Jarosch AH, Hofer M. Past and future sea-level change from the surface mass balance of glaciers. The Cryosphere, 2012, 6(6) : 1295-1322. DOI: 10.5194/tc-6-1295-2012.

[55] Radić V, Bliss A, Beedlow AC et al. Regional and global projections of twenty-first century glacier mass changes in response to climate scenarios from global climate models. Climate Dynamics, 2014, 42(1/2): 37-58. DOI: 10. 1007/ s00382-013-1719-7. 